\title{
Clinical Trials of TB Vaccines: Harmonization \& Cooperation
}

*Hassan Mahomed (South African TB Vaccine Initiative, University of Cape Town, South Africa), P. Bernard Fourie (Dept Medical Microbiology, University of Pretoria, South Africa)

*Corresponding author: Dr Hassan Mahomed

South African Tuberculosis Vaccine Initiative (SATVI),

Institute of Infectious Disease and Molecular Medicine,

University of Cape Town Health Sciences Faculty,

Anzio Rd, Observatory, Cape Town

South Africa

7925

Tel: +27 214047744 Fax: +27 214066081

Email: [hassan.mahomed@uct.ac.za]

Professor P Bernard Fourie:

Department of Medical Microbiology

University of Pretoria

Private Bag X20

Hatfield

Pretoria

South Africa

0028

Tel: +27 123192256

Email: Bernard.Fourie@up.ac.za 


\begin{abstract}
A new efficacious tuberculosis (TB) vaccine has the potential to dramatically assist control efforts for the global TB epidemic. Good progress has been made with the clinical development of new TB vaccine candidates with twelve being actively tested in clinical trials. However, there are challenges that would need to be addressed before a new vaccine is licensed for public use. The diversity of risk in populations would need to be factored into clinical development plans. Specific but feasible clinical endpoints need to be agreed upon. TB vaccines would need to be efficacious in both uninfected and infected populations. An achievable efficacy target needs to be set. Standardisation of trial outcomes and the development pipeline would need to be brought about. Alternative routes of administration should be thoroughly explored. There should be sufficient adequately prepared trial sites for doing TB vaccine assessments. Creative use of study designs should be used to expedite progress towards licensure but at the same time containing costs. There should be sufficient funding to support the TB vaccine development. These challenges can be met through commitment by role-players within the TB vaccine arena and with support by external stakeholders.
\end{abstract}

Keywords: TB vaccines; clinical trials; tuberculosis; vaccination; clinical endpoints

\title{
Introduction
}

Directly Observed Treatment Short-course (DOTS) is currently implemented in the 184 countries where, in 2006, 99\% of all estimated TB cases occurred and 93\% of the world population lived; yet Global TB incidence remains two orders of magnitude above the Stop TB Partnership goal to eliminate TB as a public health problem, defined as $<1$ case 
per million population per year by 2050. Against this background, Abu-Raddas and colleagues $^{1}$ recently argued that combining a potent new regimen of TB drugs with at least a good neonatal pre-exposure vaccine and a highly efficient molecular-based diagnostic test in a global intervention strategy can reduce annual TB incidence in 2050 by $94 \%$, over current rates. Epidemiologically, this would set the scene for elimination of TB. Other models have suggested similar strategies for achieving elimination. ${ }^{2}$

\section{The TB vaccine pipeline}

There is reason for optimism about the prospects for a new TB vaccine that is more effective than the current BCG. Fifteen new TB vaccines have been evaluated in clinical trials to date, many in high burden settings, and 12 are actively being tested currently.,4 Of these, at least 7 have entered or completed Phase IIb or Phase III testing. The development of new TB vaccines has thus progressed well, with definitive clinical assessment of different types of candidates underway. In an excellent recent review of vaccines in clinical trials, Rowland and McShane ${ }^{5}$ describe the trial approaches and status of live mycobacterial vaccines designed to replace BCG as a primary vaccination, subunit vaccines designed to enhance the effectiveness of BCG, and post-exposure therapeutic vaccines.

However, trialists face challenges. Until very recently, the collective experience with anti-TB vaccine assessment has largely been lodged in observational cohort or casecontrol studies of BCG with tuberculin skin test responses as a surrogate for immunogenicity, assuming protective efficacy, or of TB morbidity and mortality monitored over extended periods of time in large cohorts as an indicator of vaccine 
efficacy. Experiences with double-blind, randomized controlled trials to assess protective efficacy are almost non-existent, with the notable exception of the well-known Chingleput trial in India in the 1970s. ${ }^{6}$ Furthermore, regulatory experience with licensing/registration of a vaccine for tuberculosis is limited, and almost entirely focused on BCG product manufacturing and quality, and a demonstration of adequate tuberculin skin test responses as per WHO guidelines. Clearly, study designs and outcome criteria as per the BCG experience is inadequate for direct application in the assessment and selection of current candidates. However, the experience that is gradually building in the advanced clinical stage assessment of the candidates mentioned above is considerable and provides a valuable opportunity for defining standardized approaches to clinical trial design, and for establishing criteria for advancing candidates from early to final clinical phase testing. In this context, Barker, Hessel and Walker have proposed specific stagegating criteria elsewhere. [Barker et al in this issue]

Nevertheless, certain other challenges relevant to the clinical development of new vaccine candidates remain, and are briefly considered below.

\section{Diversity in risk of TB infection and disease}

Diversity in the risk of TB infection and disease and in the protective efficacy of BCG

has been observed over many decades. ${ }^{7,8}$ Specific population-associated features might result in marked differences in immune responses to the same vaccine in similar age cohorts from different parts of the world. Clearly, the clinical assessment of TB vaccines needs to take into account geographic, epidemiological variables and genetic diversity. Even smaller Phase II proof-of-concept trials may need a multi-center approach to factor 
in the impact of this diversity. More specifically, these factors include concomitant diseases with high prevalences (particularly HIV and parasitic infections), exposure to environmental mycobacteria, pre-disposing environmental conditions, socio-economic factors and population demographics. ${ }^{5}$

It is known that in the absence of an underlying HIV co-infection, the greatest proportion of TB incidence is generally driven by recent TB infections (within two years). ${ }^{9}$ There are exceptions: in young children up to the age of 2 years, and in adolescents, the progression to disease is sometimes continuous following infection, while in preadolescent years, latency is mostly maintained. Furthermore, there has been an almost universally observed higher rate of disease in males than in females in high prevalence settings in pre-HIV times; now, high rates of disease occur in co-infected females in their reproductive years. Moving forward, there is a need to re-define the clinical pathway for assessment of vaccine efficacy and the dynamic of latency and progression to disease needs to be understood for every population where vaccine trials are being run. Multicountry epidemiological cohort studies in different target age-groups are required for two important reasons: (a) To establish an adequate understanding of the epidemiology of disease pathogenesis in the absence of a new preventive intervention; and (b) To enable the design of a scientifically sound schedule for mass vaccination campaigns.

\section{Clinical endpoints}

Because of the current lack of an immune correlate of protection, efficacy trials will be dependent on clinical endpoints. These will be particularly challenging in infants because the disease is pauci-bacillary. ${ }^{10,11}$ From a vaccine development point of view, a hard 
endpoint such as bacteriological confirmation of disease is likely to be favoured by trialists, even in very young children. The proportion of sick children with bacteriologically detectable disease would be low, however, and clinical algorithms to indicate high probability for TB might be required until suitable biomarkers could be validated. It is also not possible to ignore symptoms compatible with TB in the absence of bacteriological proof, and to defer treatment. Thus, even if bacteriological proof of disease might be desired, medical interference with the development of this stage of the disease would defeat its utility as an endpoint. These special challenges for setting hard immunogenicity or vaccine efficacy targets for paediatric cohorts, indicate a need for more concerted research into what constitutes a protected or a diseased child.

In adults and adolescents, microbiological confirmation is more feasible so endpoints can more easily be defined for these groups. Nevertheless, there needs to be consensus on the definition of these endpoints in terms of the microbiological assay to be used, and the interpretation of results. Also, in the post-marketing phase, these endpoint definitions need to be such that outcomes could reasonably be observed and documented by TB control programme staff and primary health care service providers. The development and integration of modern molecular diagnostic procedures for the detection of the pathogen, and of any relevant assays to detect enhanced immunogenicity, is necessary especially as part of a pre-defined set of tests to be used as part of ongoing vaccine safety and efficacy assessments. 


\section{What level of efficacy will be acceptable for a new TB vaccine?}

A range of protective efficacies between $60 \%$ and $90 \%$ have been proposed in an opinion survey conducted amongst TB vaccine experts (as part of the development of the $T B$ Vaccine Blueprint) as necessary for licensure of a TB vaccine. The lack of consensus highlights the importance of this issue, and might have implication for mass introduction of a vaccine in populations where the benefits are potentially great. Lessons can be learned from the malaria field, where the acceptance of efficacy levels of 53\%-65\% in phase II trials for a malaria vaccine were regarded as sufficient for moving forward into a phase III trial. ${ }^{12,13}$ A number of factors will influence discussions about an acceptable level of efficacy for TB vaccines, however. One factor is the variable efficacy of BCG against pulmonary $\mathrm{TB}$, making it difficult to set a value for relative efficacy of a new vaccine versus BCG, and might require an absolute level to be stated. Another key question is what level of efficacy has both a significant public health impact and a compelling cost/benefit ratio. Mathematical modeling may help to answer these questions and to determine the impact of vaccination in selected populations. Modelling data suggest that a $60 \%$ level of efficacy is likely to have a substantial public health impact. ${ }^{1,2}$ TB is a massive public health problem and the impact of fairly modest gains in efficacy over BCG by next generation vaccine candidates should not be underestimated.

\section{Standardisation}

Standardization of parameters and definitions to be used in the safety assessment of all $\mathrm{TB}$ vaccine candidates is a high priority. In this regard, the work of groups such as the Brighton collaboration (https://brightoncollaboration.org/public) may be useful. 
Likewise, standardization of the criteria to be met in the clinical development pathway, is essential. Some vaccines have gone through a gradual age de-escalation path from adults to adolescents to children to infants whilst others have been able to move directly from adult to infant studies. This partly reflects an evolution in regulatory requirements among different regulatory authorities. Not all vaccines in Phase IIb proof-of-concept trials have undergone interference assessments with other childhood vaccines, for example. This is an important step in planning towards implementation where in all likelihood a licensed efficacious vaccine would best be given with other vaccines. Safety in the HIV-infected community (adults and exposed infants) is crucial, and all vaccines should be required to be tested for safety in this group, given the high prevalence HIV in a number of high TB burden countries.

\section{Alternative routes of vaccination}

Preparedness for clinical assessment of vaccines delivered by alternative routes (e.g pulmonary, nasal, oral) might also be required, given recent developments in this context. ${ }^{14}$ The lung is the primary, if not the sole, portal of entry for mycobacteria that cause TB, and it has been of interest since the 1950s to deliver certain key drugs and the TB vaccine BCG by the same route. Because of technical difficulties, also for pharmaceutical reasons, most of these early studies were abandoned despite encouraging results. In recent years, however, a renewed interest in formulating vaccines for pulmonary delivery emerged, for reasons that remain significant. Targeting the lung mucosa for immunisation against TB potentially offers advantages over current injection approaches, with advanced studies in primates with new subunit candidates and BCG, 
showing promising results. ${ }^{15}$ Such an approach might be particularly suitable for live recombinant or attenuated vaccine candidates.

The nasal route of administration has been investigated for flu vaccines and might offer lessons for TB vaccine development, in particular for adjuvanted or viral vectored candidates.

Several recent investigations involving room-temperature stable oral microparticle preparations of BCG show promising results in bovine models, and this route of delivery might hold promise for application in humans as well. ${ }^{5,16}$

Although not yet having been explored in the context of TB vaccines, skin patches containing microneedles coated with dried solutions of various other vaccines, including influenza vaccines, also hold promise as needle-free options. These are early developments worthy of further investigation.

The emphasis for changing the route of delivery would be on developing needle-free, room temperature stable vaccine products that would be easy and cheap to administer, and would enhance a protective immune response by targeted delivery to appropriate immunological compartments. Recent publications suggest that such technologies are within reach, and would be affordable and pharmaceutically scaleable. ${ }^{17}$

\section{Trial sites}

Given the fact that BCG has in some populations and age cohorts managed to reach a protective efficacy of around $80 \%$, there is little scope for any vaccine to do much better in similar target groups, and large numbers of subjects would need to be enrolled in order 
to demonstrate better efficacy. In such situations, prime-boost studies with the objective of showing significantly better long-term protection, rather than BCG-replacement studies, might be the objective.

Populations with the highest prevalence of disease would be chosen for establishing vaccine trial sites, and such efforts are currently being widely pursued in areas like subSaharan Africa where TB rates are fuelled by the high prevalence of HIV co-infection. Trial capacities would need to be integrated (but not necessarily merged) in a targeted way, in order to access large populations at risk of disease so that small margins of improved vaccine efficacy could be detected. Accurate estimates of incidence rates are key to planning efficacy trials. This may be challenging in certain areas where only routine data are available. In this regard, routinely collected TB control programme data often underestimate true incidence, in particular as far as case definitions compatible with clinical trial endpoints are concerned. Data from specially-conducted epidemiological studies might be required.

Another reasonable approach might be to define large, global networks that would aim to conduct only specific types of studies. There are essentially three arenas for Phase II/III trials: (A) Live mycobacterial vaccines designed for replacing BCG; (B) Subunit vaccines designed to boost BCG; and (C) Therapeutic vaccines designed as an adjunct to chemotherapy. For A, strong capability in paediatric TB research and health service delivery is essential. Target cohorts would be infants and young children, including babies born from HIV-infected mothers. Trialists, including vaccine developers, would need to gain consensus on which endpoints to use and would likely have the need for access to specialised clinical laboratory and radiological infrastructure. 
For B, access to adolescent and adult populations is important. This is likely to be the largest but also most challenging population for the immediate future (those requiring HPV vaccination are similarly challenging). Significant networking across diverse geographical areas are beginning to be utilized for certain advanced TB vaccine candidates, but may not be accessible to later vaccines candidates now transitioning into Phase II studies. A very comprehensive networking effort is called for in order to allow for sufficiently powered studies to be conducted. In these cohorts, disease as detected microbiologically, is a reasonable indicator of vaccine efficacy, and a rapid diagnostic infrastructure to detect the pathogen is essential. Populations with substantial prevalences of latent TB infection would be needed since proposed mass campaigns currently being proposed as a strategy with a successful vaccine would be done in individuals with and without latent infection.

For $\mathrm{C}$ the aim will be to provide access to special risk groups with high rates of $\mathrm{TB}$, and where the risk of disease progression from an underlying TB infection would be high. A history of BCG vaccination in these groups is typically remote. Subjects for recruitment might include TB patients on treatment, or TB-HIV co-infected adults on preventive isoniazid therapy. A close interaction with TB drug research groups might be useful.

\section{Target groups}

Although clinical development to date has shown that the candidate TB vaccines are by and large safe (in the groups tested) and provoke a range of immune responses, there is, as yet, no unequivocal indication of vaccine efficacy, although some candidates clearly have demonstrated promise in phase II and III trials. Phase IIb trials are in progress in 
infants and HIV positive adults with efficacy objectives. However, given the challenges of demonstrating efficacy in these two target groups, sponsors of vaccines at an earlier stage of development, may follow an approach of doing efficacy trials in HIV negative adolescents/adults to confidently assess vaccine efficacy. Vaccine candidates thus achieving measured efficacy may then be subsequently introduced to infant and HIV positive cohorts for vaccine efficacy assessments. The discovery of immune correlates of protection would help expedite the length and costs of such trials.

Vaccines targeted at adolescents/adults need to be versatile and be able to prevent infection as well as reactivation in latently infected individuals. In particular, safety and efficacy in HIV co-infected individuals will be important. Currently, such trials are being conducted in high co-infection prevalence settings in South Africa, which should guide the way for trial designs appropriate to other candidates. It is anticipated that subunit vaccines or non-replicating whole-cell constructs would be eligible vaccine candidates in this context.

\section{Study designs and trial costs}

Some degree of creativity in designing Phase III trials for assessing vaccine efficacy is clearly required, with the key points to keep in mind being as follows:

- The role of BCG in enhancing or inhibiting protective immune responses is unknown - it is assumed to be enhancing. Evidence of efficacy will be needed in order to develop immunological correlates (surrogates) of protection that can be used to determine immune-protection rates and level of immunity in vaccinees. 
- From a regulatory point of view, the concept of a "Phase IIb/III" type trial needs to be explored with regulatory authorities who may find such an approach challenging.

Clinical trial applications that intend to permit amendments to the protocol should prospectively include a description of the intended change points, the arrangements for independent interim analysis that will drive such changes, and the mechanisms that will ensure statistical validity of the results. Oversight by Data and Safety Monitoring Boards (DSMBs), or similar independent safety committees, will be required.

Head-to-head comparisons of new TB vaccines have not occurred to date in human trials, but have occurred with other vaccines such as pertussis, and can serve as a model for similar trial designs with different TB candidates. The benefits of head-to-head comparisons include, firstly, the reduction of costs of clinical trials if a single protocol tests a number of vaccines simultaneously at a single site. There is no reason why early phase trials cannot be conducted in this way. Secondly, this may be an important step for deciding which vaccine or vaccines proceed to the next stage of development. Thirdly, this will clearly be one way in which issues of standardization may be addressed. However, vaccine developers may be resistant to this idea for various reasons such as protection of their intellectual property. Therefore, contractual arrangements with multiple partners may be complex. Also, if these trials are performed at early phases, one would be dependent on immunogenicity measurements as a proxy to determine potential efficacy and there is not enough scientific evidence to support such an approach. The fact that new TB vaccines are at different stages of development will also make head to head comparisons difficult. 
Moving towards efficacy trials which require larger subject numbers and are therefore more expensive, there is recognition of the fact that funding for such trials will be limited. There would need to be a rational approach for selecting candidates for efficacy trials, as clearly, there will not be enough funding for all candidates to undergo such trials. [see Barker et al in this series]

On the other hand, there should be an exploration of reducing the costs of such trials. One suggestion is to utilize existing trial site networks such as the HIV Vaccines Trial Network (HVTN) and IMPAACT to mitigate the costs of setting up suitable trial sites. Another way of reducing costs is through creative trial designs. Simplifying follow-up or using existing infra-structure such as demographic surveillance sites or routine health care services are options that should be considered. However, trial designs need to factor in meeting the required regulatory standards while trying to deal with costs constraints. Partnering with large pharmaceutical companies will be critical in addressing some of the cost constraint issues. Others may be adaptive trial designs, where multiple candidates are studied simultaneously and the expected failure of some vaccines which do not meet the stage-gate criteria, should also be evaluated as cost-saving options. Another cost-saving approach is to use a stepped-wedge design in Phase III studies where one vaccine (e.g. BCG) is replaced by the new vaccine in different arms at different time-points. This approach has been successfully used in a hepatitis intervention study in the Gambia ${ }^{18}$ and might offer a model for vaccine candidates proposed to replace BCG. 


\section{Conclusion}

In conclusion, good progress has been made in the clinical development of new TB vaccines. There are many challenging areas that need to be addressed such as trial designs, funding, alternative routes of vaccination, TB epidemiology and standardization of outcome measures and clinical pathway. However, with commitment and resources to TB vaccines, a promising avenue for global TB control may achieve substantial success.

\section{Acknowledgements}

We wish to thank Michael Brennan (Aeras), Jelle Thole (TuBerculosis Vaccine Initiative), and Chris Dye (World Health Organization) for valuable comments on the manuscript.

\section{References}

1. Abu-Raddad LJ, Sabatelli L, Achterberg JT, Sugimoto JD, Longini IM, Jr., Dye C, et al. Epidemiological benefits of more-effective tuberculosis vaccines, drugs, and diagnostics. Proc Natl Acad Sci U S A 2009;106:13980-5.

2. Young D, Dye C. The development and impact of tuberculosis vaccines. Cell 2006; 124:683-687.

3. Stop TB Working Group on TB vaccines. Tuberculosis Vaccine Candidates - 2010. http://www.stoptb.org/wg/new_vaccines/assets/documents/TB\%20Vaccine\%20Pipeli ne\%2010\%20-\%2003\%2021\%2011.pdf accessed on 18 November 2011.

4. Hawkridge T, Mahomed H. Prospects for a new, safer and more effective TB vaccine. Paediatr Respir Rev 2011;12:46-51. 
5. Rowland R, McShane H. Tuberculosis vaccines in clinical trials. Expert Rev. Vaccines 2011;10:645-658

6. Tripathy SP. Fifteen year follow-up of the Indian BCG prevention trial. Bull. Int. Union Tubercle Lung Dis. 1987;62:69-72.

7. Fine PE. Variation in protection by BCG: implications of and for heterologous immunity. Lancet 1995;346:1339-45.

8. Rodrigues LC, Diwan VK, Wheeler JG. Protective effect of BCG against tuberculous meningitis and miliary tuberculosis: a meta-analysis. Int J Epidemiol 1993;22:1154-8.

9. Sutherland I, Svandova E, Radhakrishna S. Alternative models for the development of tuberculosis disease following infection with tubercle bacilli. Bull Int Union Tuberc 1976;51:171-9.

10. Mulenga H, Moyo S, Workman L, Hawkridge T, Verver S, Tameris M, et al. Phenotypic variability in childhood TB: implications for diagnostic endpoints in tuberculosis vaccine trials. Vaccine 2011;29:4316-21.

11. Hatherill M, Verver S, Mahomed H. Consensus Statement on Diagnostic EndPoints for Infant Tuberculosis Vaccine Trials. Clinical Infectious Diseases 2011; In press.

12. Asante KP, Abdulla S, Agnandji S, Lyimo J, Vekemans J, Soulanoudjingar S, et al. Safety and efficacy of the RTS,S/AS01E candidate malaria vaccine given with expanded-programme-on-immunisation vaccines: 19 month follow-up of a randomised, open-label, phase 2 trial. Lancet Infect Dis 2011;11:741-9.

13. Aponte JJ, Aide P, Renom M, Mandomando I, Bassat Q, Sacarlal J, et al. Safety of the RTS,S/AS02D candidate malaria vaccine in infants living in a highly endemic 
area of Mozambique: a double blind randomised controlled phase I/Ilb trial. Lancet 2007;370:1543-51.

14. Fourie PB, Germishuizen WA, Wong YL, Edwards DA. Spray drying TB vaccines for pulmonary administration. Expert Opin Biol Ther 2008;8:857-63.

15. White AD et al. An evaluation of the mucosal immunogenicity of BCG prime / mucosal boost vaccine regimens in macaques. Poster presentation, Second Global Forum on TB Vaccines. Tallinn, Estonia; 2010.

16. Aldwell W. An oral delivery system for targeting TB vaccines to the mucosal immune system. In: Second Global Forum on TB Vaccines. Tallinn, Estonia; 2010.

17. Garcia-Contreras L, Wong YL, Muttil P, Padilla D, Sadoff J, Derousse J, et al. Immunization by a bacterial aerosol. Proc Natl Acad Sci U S A 2008;105:4656-60.

18. The Gambia Hepatitis Intervention Study. The Gambia Hepatitis Study Group. Cancer Res 1987;47:5782-7. 